\title{
SI for
}

\section{Assessing sub-grid variability within satellite pixels using airborne mapping spectrometer measurements}

Tang et al., 2020
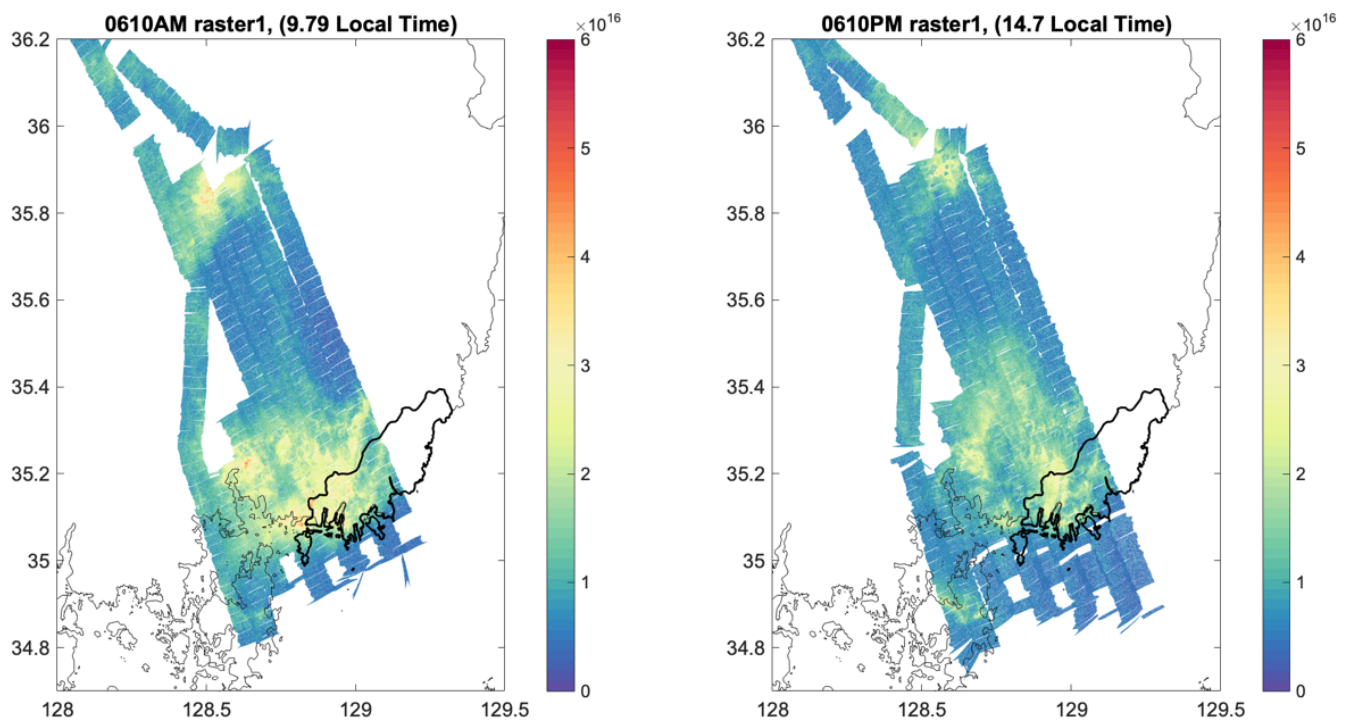

Figure S1. GeoTASO data of tropospheric $\mathrm{NO}_{2} \mathrm{VC}$ (molecules $\mathrm{cm}^{-2}$ ) measured during KORUSAQ over the Busan region. Each panel shows a separate raster. Panel titles show month, day, $\mathrm{AM} / \mathrm{PM}$, raster number on that date, and mean time of raster acquisition. 

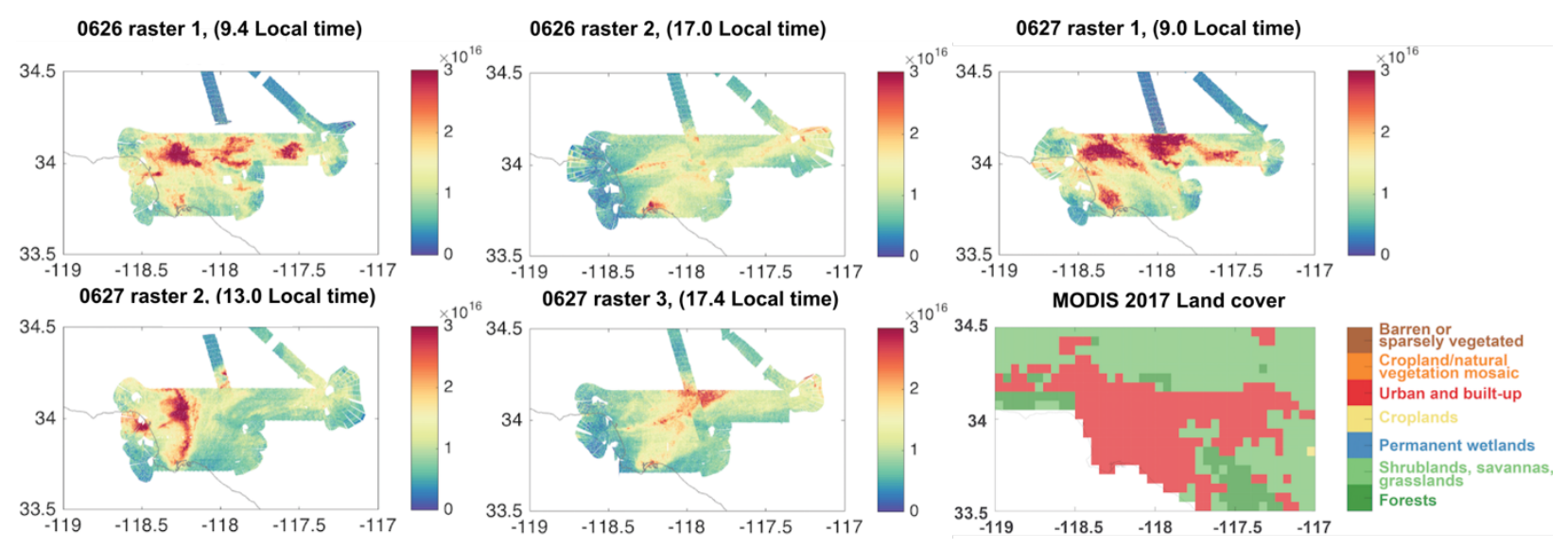

Figure S2. GeoTASO data of tropospheric $\mathrm{NO}_{2} \mathrm{VC}$ (molecules $\mathrm{cm}^{-2}$ ) measured during the SARP 2017 campaign over the Los Angeles (LA) Basin. Top panels and lower left-most two panels show separate rasters; panel titles show month, day, raster number on that date, and mean time of raster acquisition. Bottom right panel shows land cover map over the LA basin domain (MODIS Terra and Aqua MCD12C1 L3 product, version V006, annual mean at $0.05^{\circ}$ resolution; Friedl et al., 2015). 

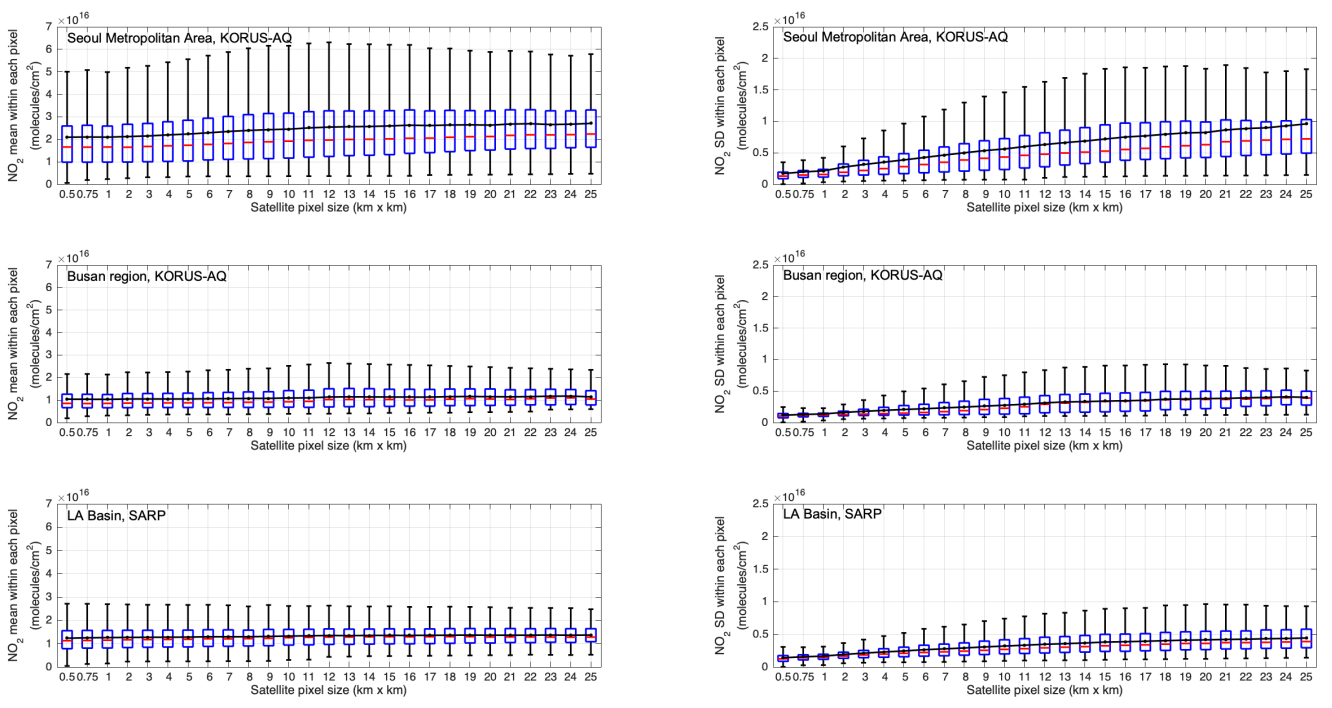

Figure S3. Boxplot for the mean (molecules $\mathrm{cm}^{-2}$ ) (panel a, c, e) and standard deviation (molecules $\mathrm{cm}^{-2}$ ) (panel b, d, f) within hypothetical satellite pixels over the Seoul Metropolitan Area, Busan region, and Los Angeles Basin. Medians are represented by red bars, interquartile ranges between 25 th and 75th percentiles by blue boxes, and the most extreme data points not considered outliers by whiskers. The black lines represent the average of the mean and SD within satellite pixels at a given size. 

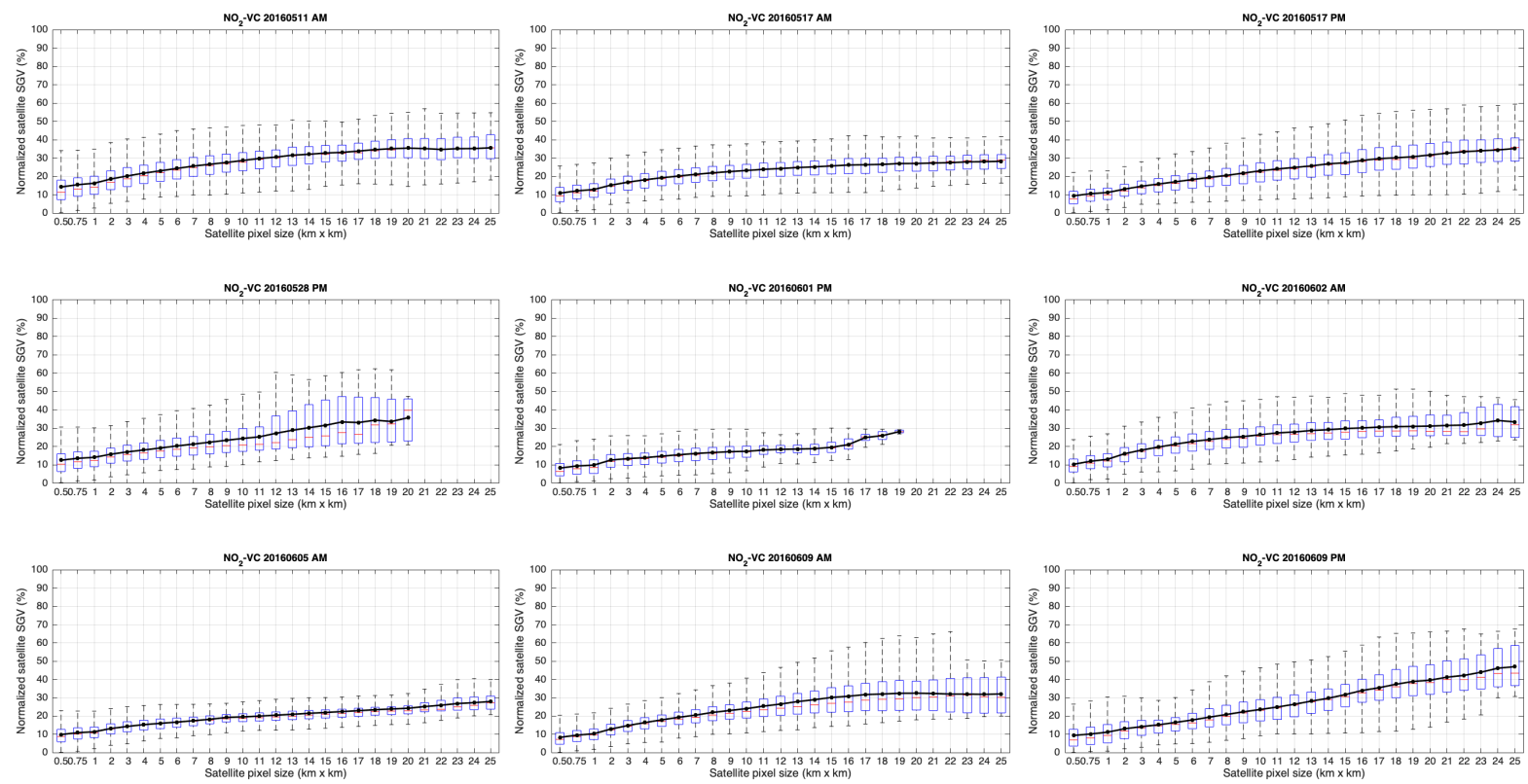

Figure S4. Same as Figure 4 but for individual flights over SMA. 

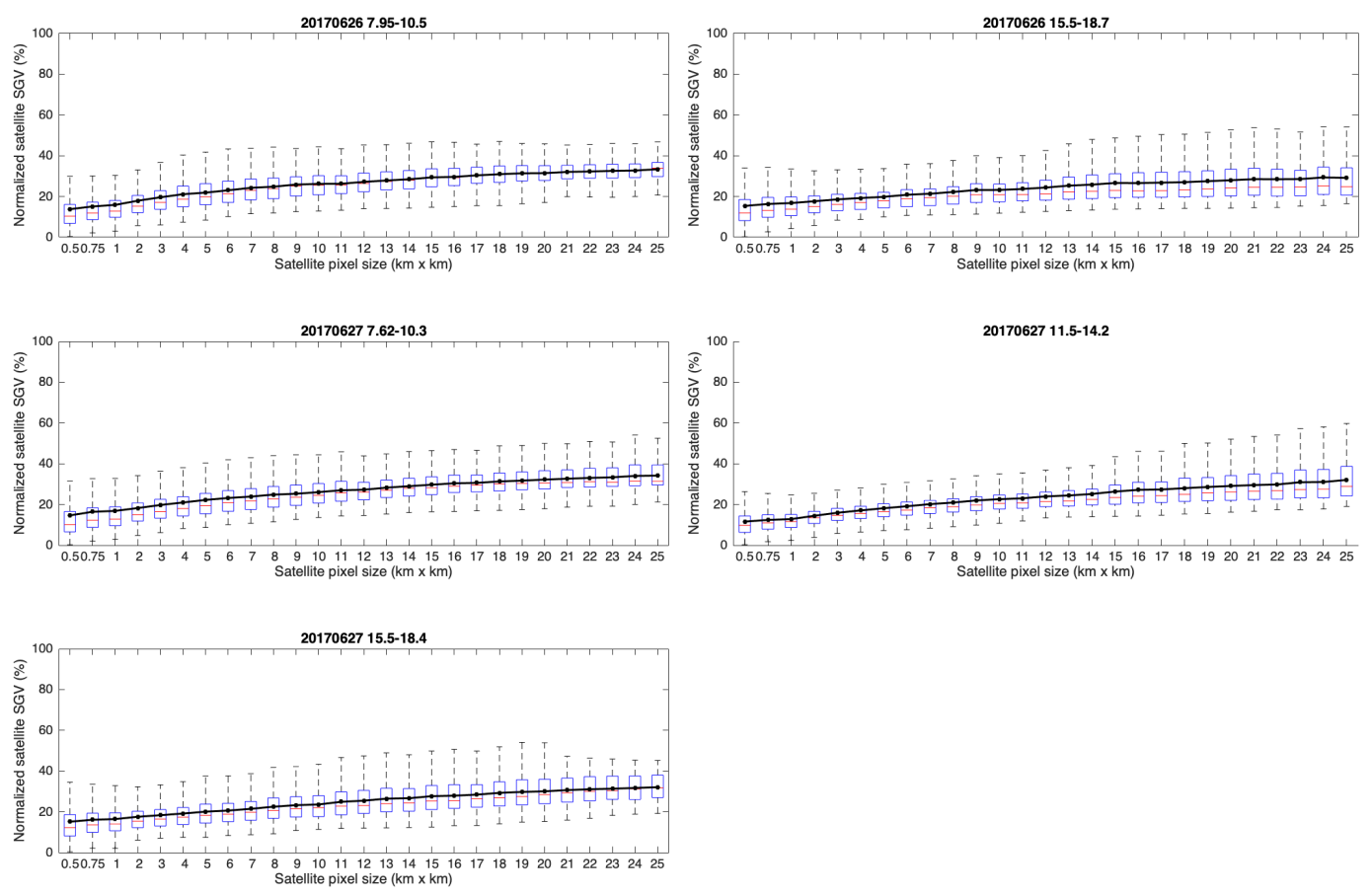

Figure S5. Same as Figure 4 but for individual flights over LA Basin. 


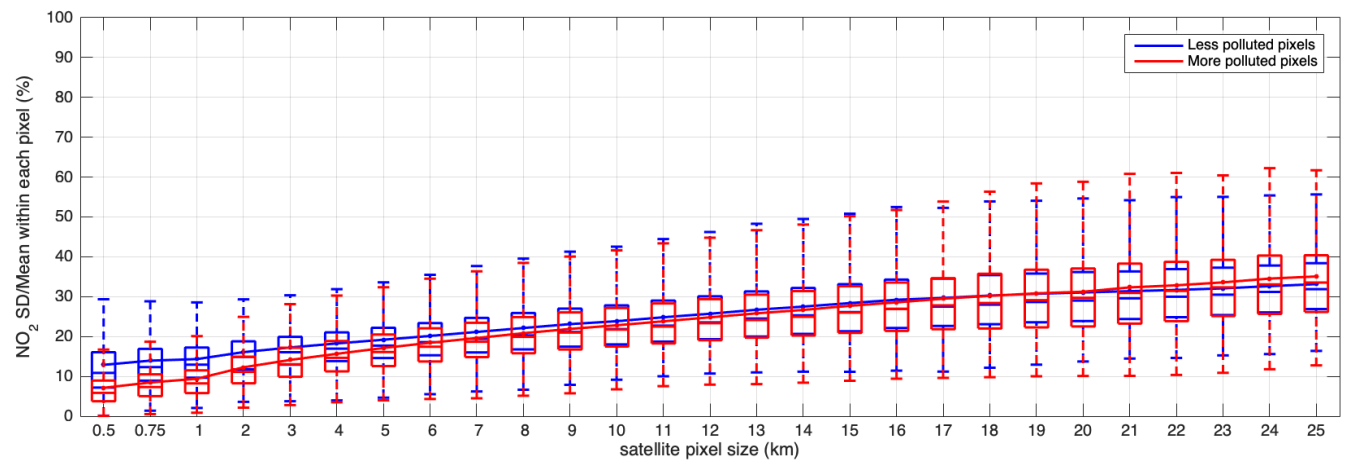

Figure S6. Boxplot (with medians represented by bars, interquartile ranges between 25 th and 75 th percentiles represented by boxes, and the most extreme data points not considered outliers represented by whiskers) for the relative sub-grid variabilities within hypothetical satellite pixels that are less polluted (the mean of the satellite pixel is lower than the average value of all pixels, i.e., $2 \times 10^{16}$ molecules $\mathrm{cm}^{-2}$; shown in blue) and more polluted (the mean of the satellite pixel is higher than the average value of all pixels, i.e., $2 \times 10^{16}$ molecules $\mathrm{cm}^{-2}$; shown in red). 

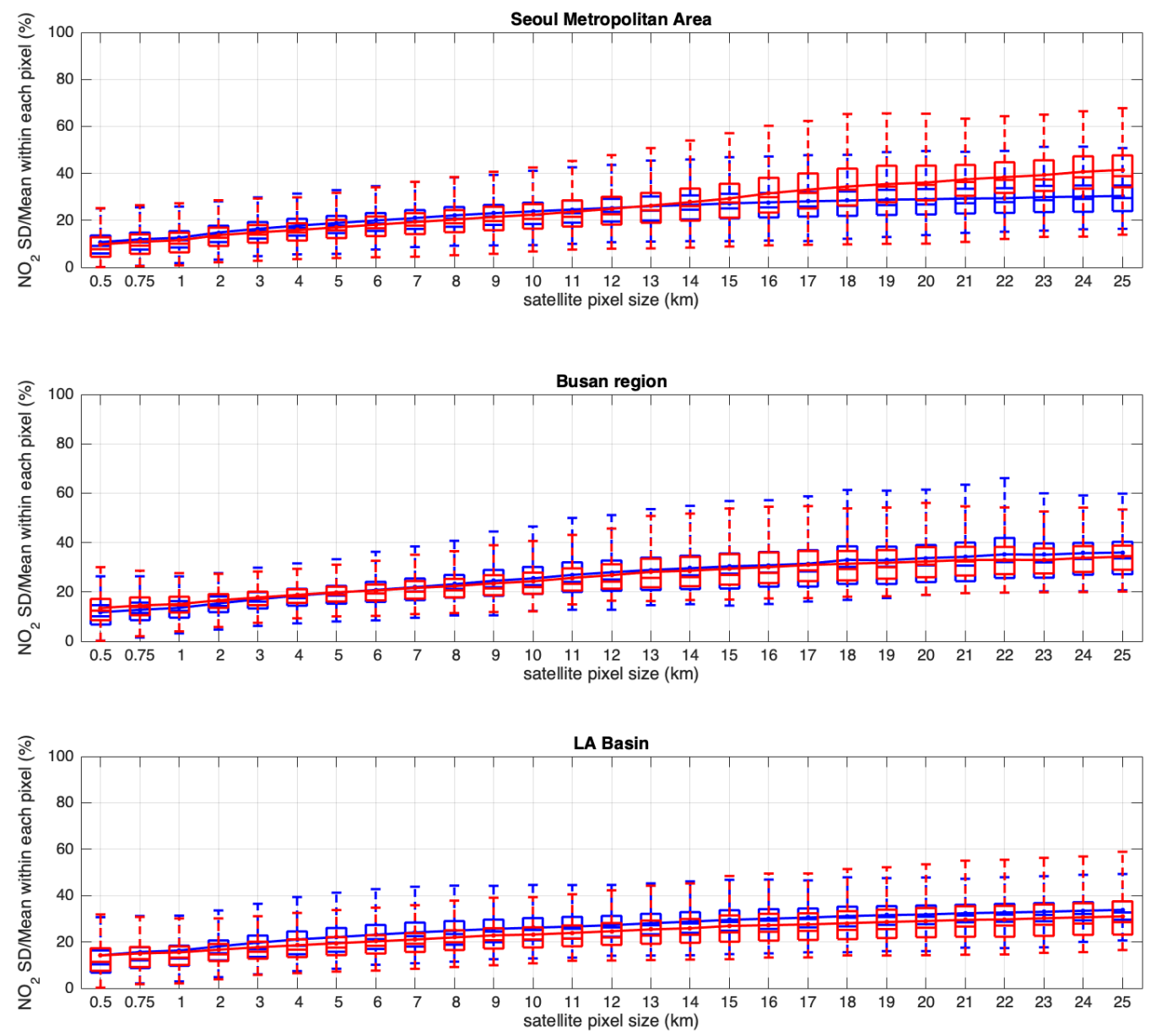

Figure S7. Boxplot (with medians represented by bars, interquartile ranges between 25 th and 75 th percentiles represented by boxes, and the most extreme data points not considered outliers represented by whiskers) for the relative sub-grid variabilities within hypothetical satellite pixels over the Seoul Metropolitan Area, Busan region, and Los Angeles Basin, sampled over morning (blue) and afternoon (red) GeoTASO data. The blue/red dots and the blue/red line represent the mean of the relative sub-grid variabilities within satellite pixels at a given size sampled in the morning/afternoon. 

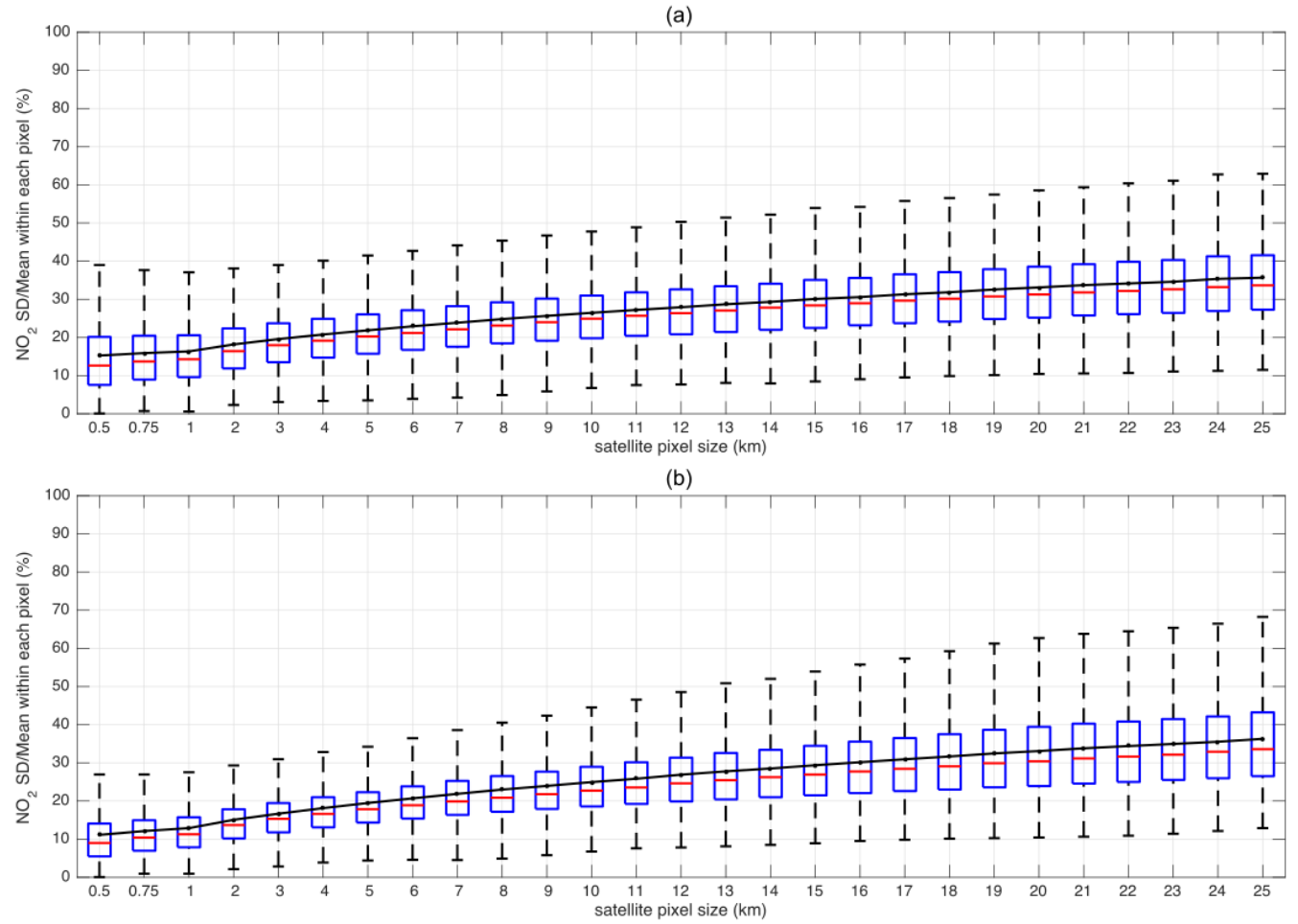

Figure S8. Same as Figure 4 but for (a) sampling hypothetical satellite pixels directly without grouping the GeoTASO data by time over SMA and (b) sampling hypothetical satellite pixels based on rasters. 


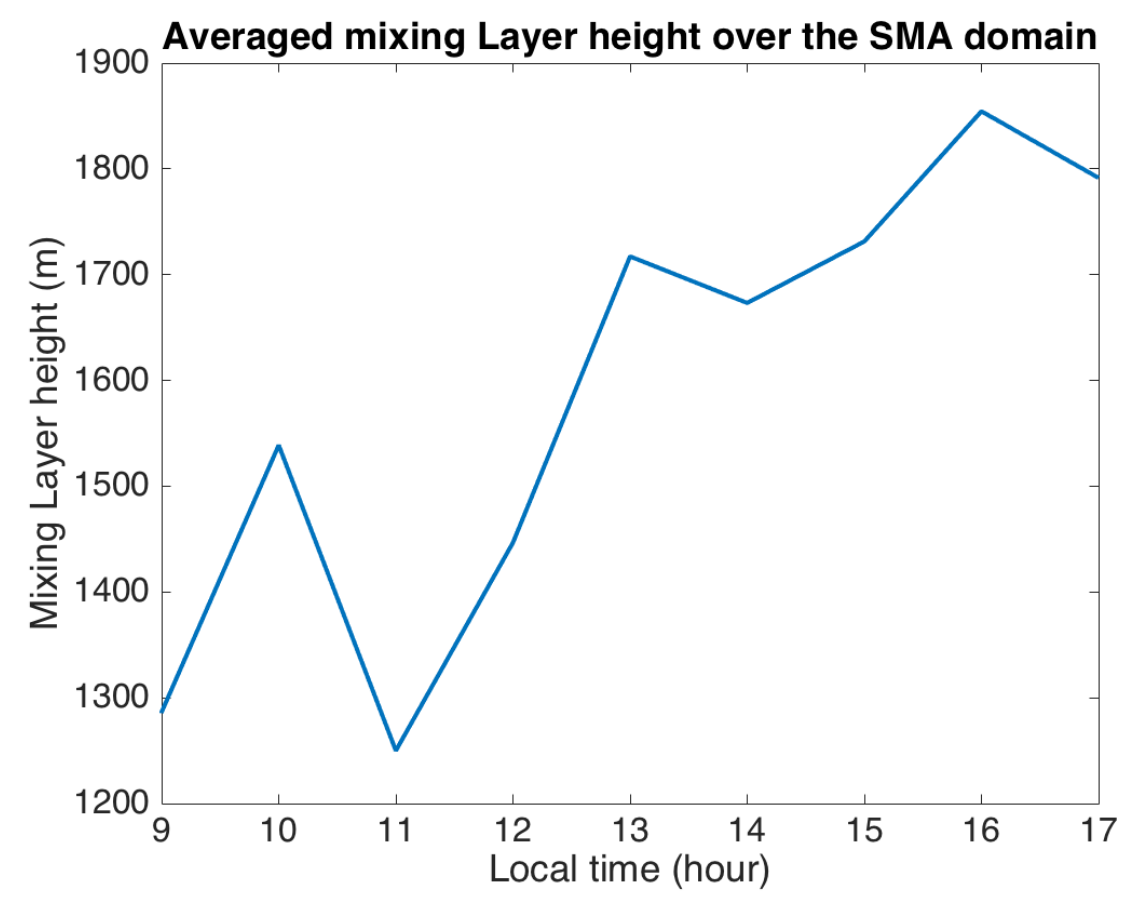

Figure S9. Averaged hourly mixing layer height over the Seoul Metropolitan Area during KORUS-AQ. The mixing layer height data are derived from the KORUS-AQ Airborne Differential Absorption Lidar - High Spectral Resolution Lidar (DIAL-HSRL) measurements of aerosol backscatter. Mixing layer height is only approximately equal to planetary boundary layer (PBL) height. 


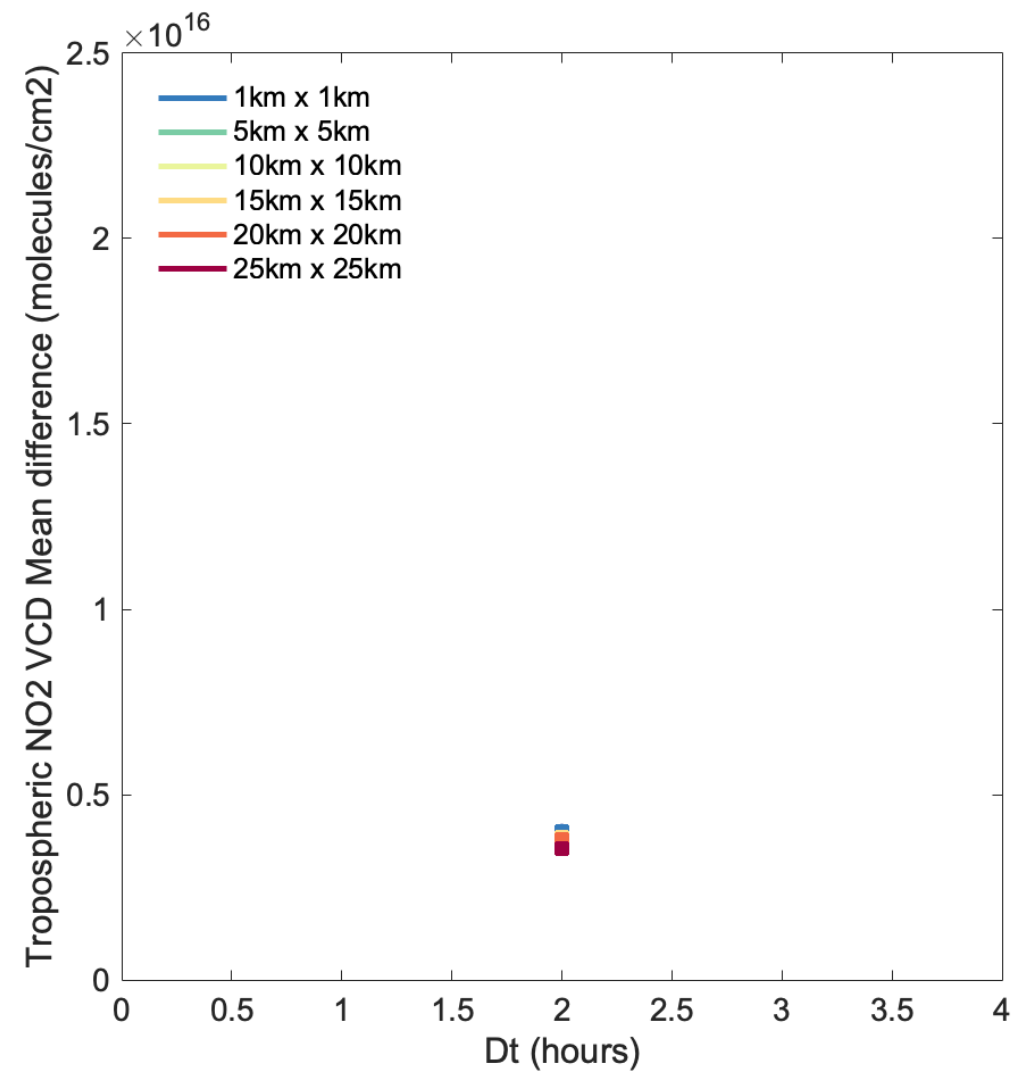

Figure S10. Same as Figure 6 but for Busan region. 


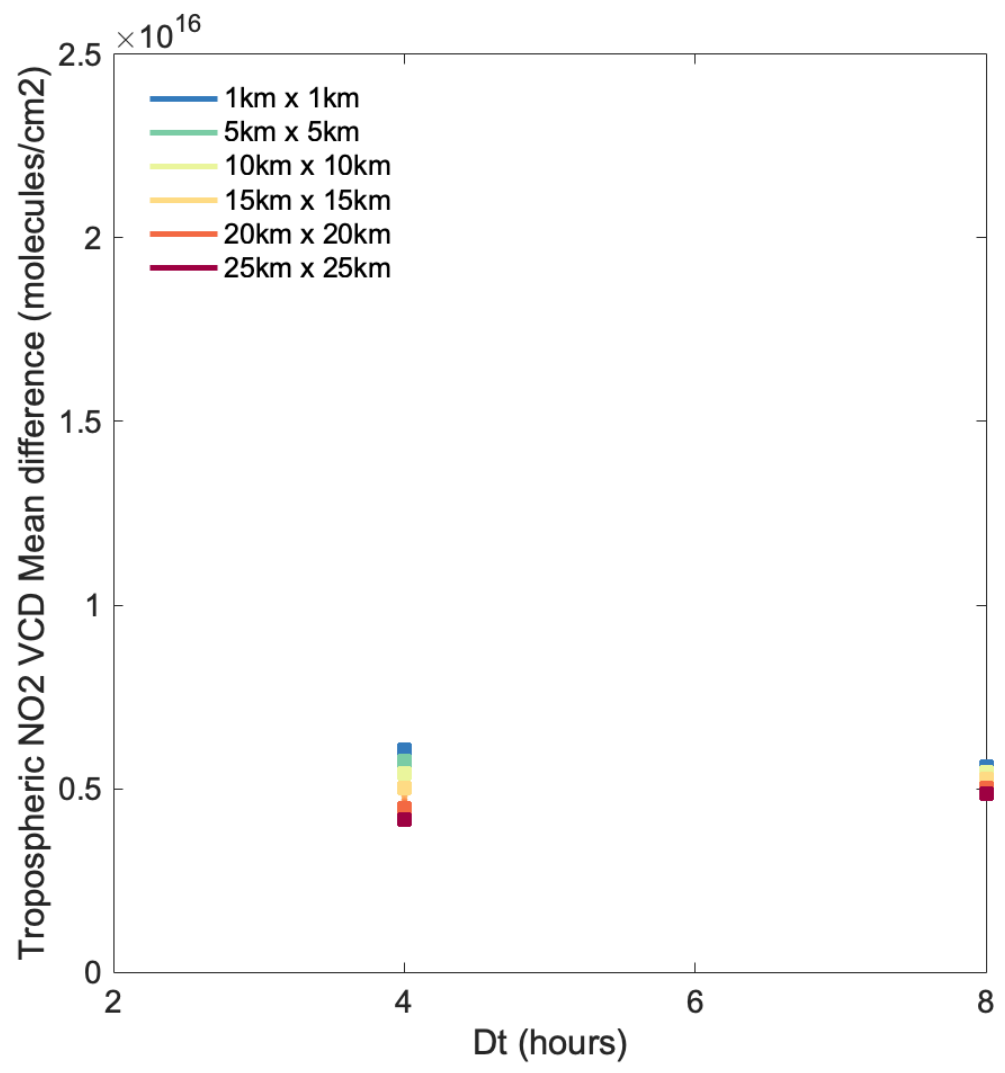

Figure S11. Same as Figure 6 but for Los Angeles (LA) Basin. 


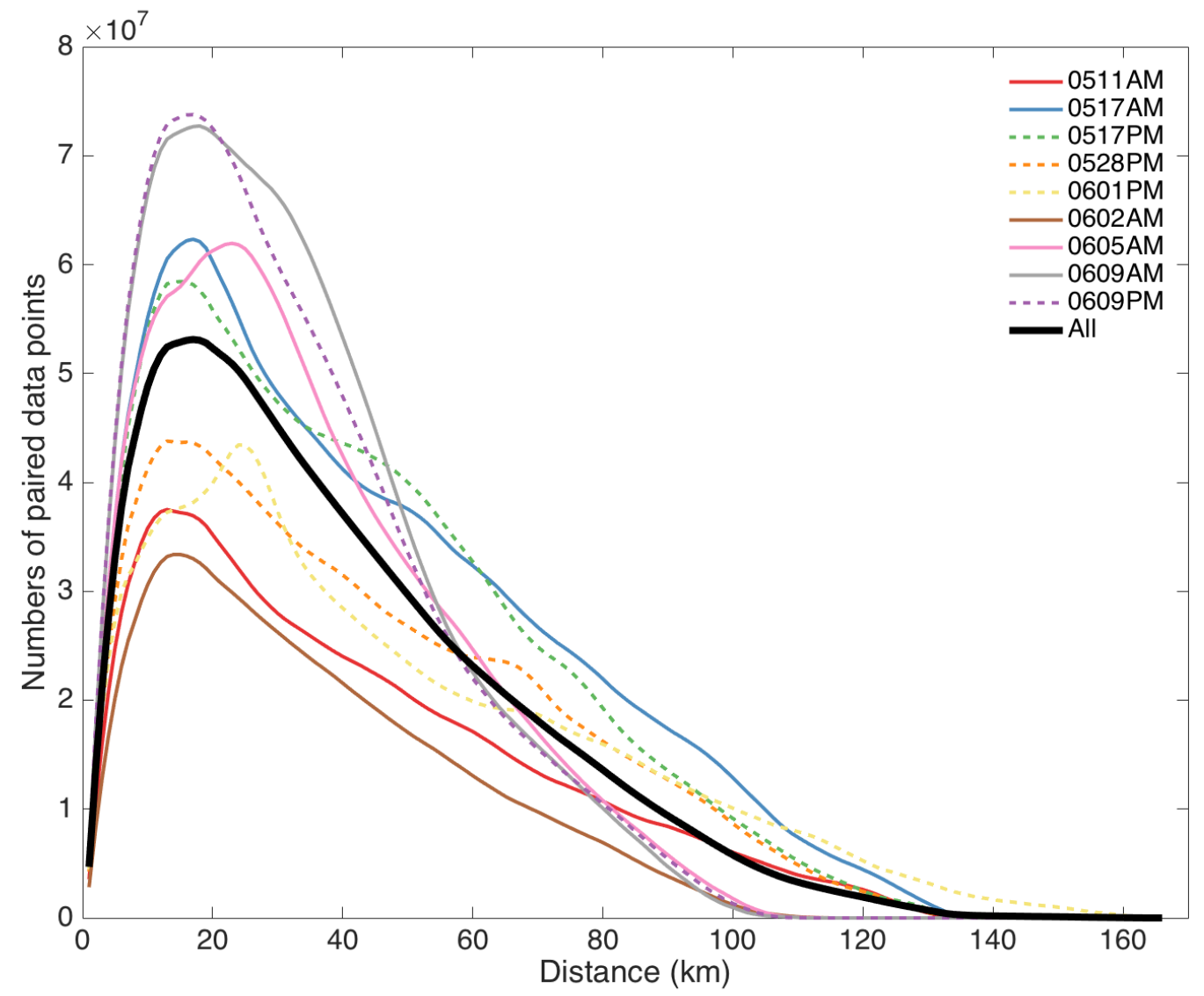

Figure S12. Numbers of paired GeoTASO data points used to calculate Spatial Structure Function (SSF; Figure 7) over the Seoul Metropolitan Area (SMA) during KORUS-AQ. 

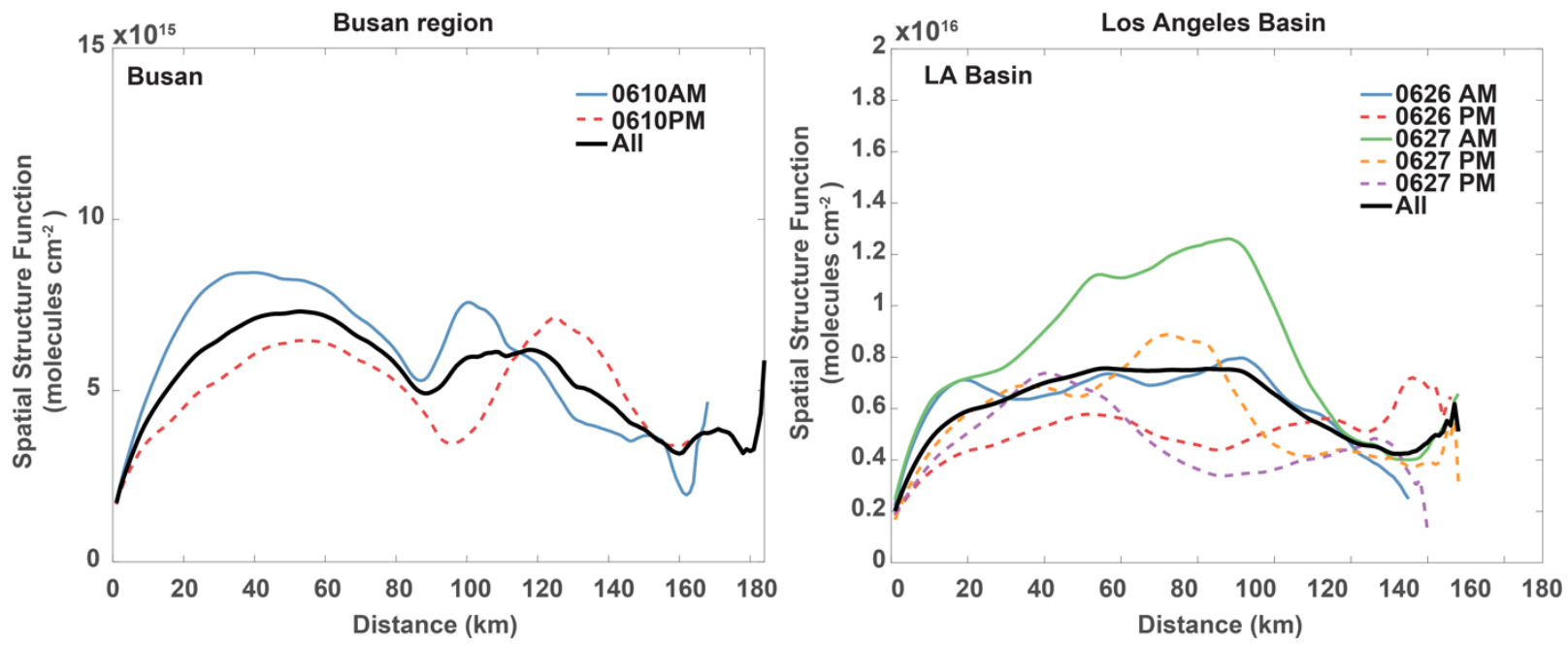

Figure S13. Same as Figure 7a but for Busan (left) and Los Angeles (LA) Basin (right). 

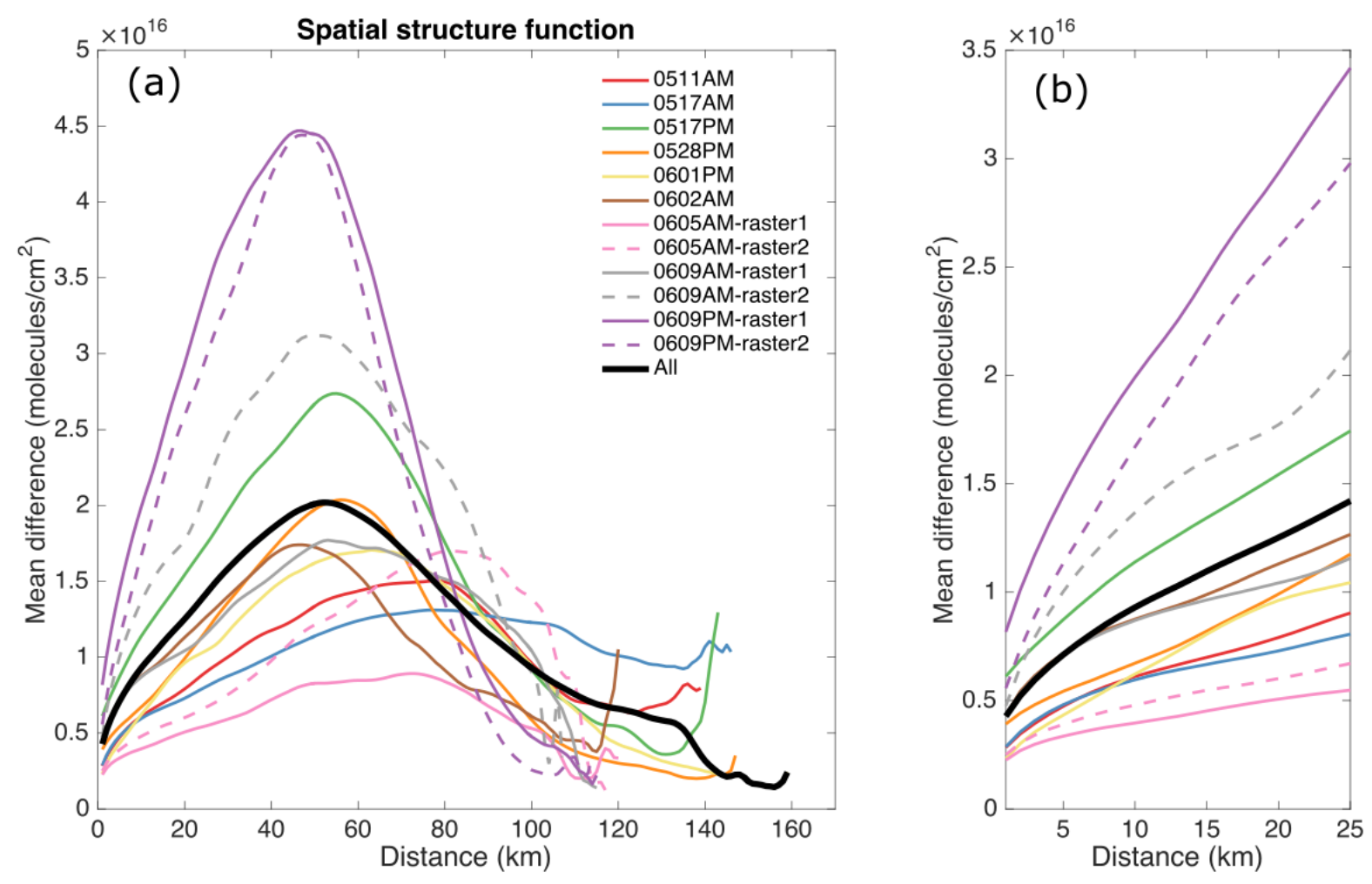

Figure S14. (a) Spatial Structure Function (SSF) for GeoTASO data of tropospheric $\mathrm{NO}_{2}$ VC molecules $\mathrm{cm}^{-2}$ ) over the Seoul Metropolitan Area (SMA) during KORUS-AQ and (b) the zoomin version of panel (a) for distance range of 1-25 km. The SSF calculates average of absolute value of $\mathrm{NO}_{2, \mathrm{VC}}$ differences (i.e., mean difference; y-axis) across all data pairs with in the same raster (instead of hourly bin) that are separated by different distance (y-axis). The SSF based on GeoTASO data measured during morning flights are in solid colored lines while the SSF based on GeoTASO data measured during afternoon flights are in dashed colored lines. The SSF based on all the data are in black solid line. 

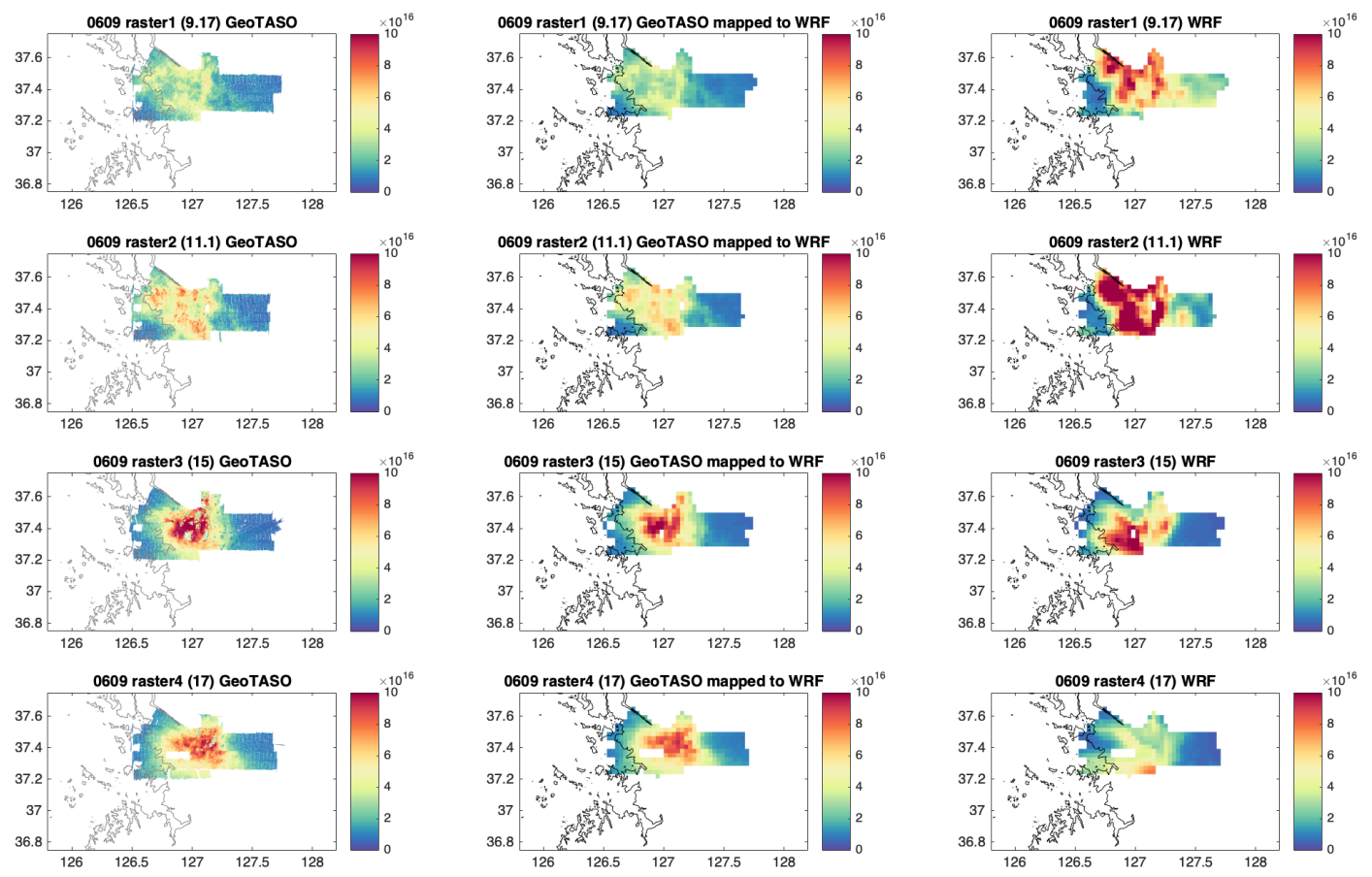

Figure S15. GeoTASO data of tropospheric $\mathrm{NO}_{2}$ vertical column (molecules $\mathrm{cm}^{-2}$ ) measured during 4 rasters on June $9^{\text {th }}, 2016$ over the Seoul Metropolitan Area (left column), GeoTASO data gridded to WRF-Chem grids (middle column), and corresponding filtered WRF-Chem model output according to the GeoTASO data coverage (right column). 